\title{
Kleine-Levin syndrome: a cause of diagnostic confusion
}

\author{
M Pike, G Stores
}

\begin{abstract}
The case is described of a boy with the Kleine-Levin syndrome in whom prominent behavioural disturbances and the initial absence of a clear cyclical pattern obscured the diagnosis. Treatment with lithium was effective.

(Arch Dis Child 1994; 71: 355-357)
\end{abstract}

The Kleine-Levin syndrome is a rare disorder of uncertain aetiology, typically with onset in adolescence and a tendency to spontaneous remission. ${ }^{1}$ Its essential feature is episodic hypersomnia, but the behavioural disturbances which commonly accompany this sleep disorder can be so prominent that they may well lead to an erroneous diagnosis of a primarily psychological disorder.

\section{Case history}

The patient was a 9 year old boy with no significant previous medical or psychiatric history apart from minor reading difficulties. In August 1991 he slipped while in a sports centre and fractured his left radius. Over the subsequent four days he required three general anaesthetics for orthopaedic procedures without any complication. He was discharged home five days after admission, when he was noted to be rather sleepy. Ten days later, he was still rather sleepy and had developed headaches and vomiting. He was treated for tonsillitis with co-trimoxazole by mouth.

A few days later he became disinhibited, overactive, aggressive, and hyperphagic, with immature speech. He was admitted to hospital where computed tomography of his brain and cerebrospinal fluid studies were normal. Empirical treatment was started with intravenous acyclovir for three days, followed by acyclovir by mouth for a further seven days. Ten days after admission his behaviour was more or less normal and he was discharged home. A day or two later, however, when he returned to school, his behaviour deteriorated again.

At this stage, the boy was admitted to his regional centre for further assessment. He was noted to be aggressive and disinhibited with obscene language, disorientated in time, and perseverative in his speech. At times he was noted to be abnormally drowsy as well as disinhibited. As his behaviour and alertness fluctuated day by day, the question of substance abuse was raised.

Extensive investigations for a biochemical, metabolic, endocrine, inflammatory, toxicological, or epileptic disorder were normal.
After psychiatric assessment it was suggested that there was probably a psychological explanation for the boy's disturbed behaviour. His mother was unhappy with this suggestion and arranged discharge.

Some weeks later, admission was arranged to another regional centre. By this time, a clearly cyclical pattern had emerged to his behavioural disturbance; good and bad episodes occurred, each lasting about a week. During a good episode, the boy was his usual pleasant self, with normal appetite and sleep patterns. During a bad period he was truculent, monosyllabic, slow to respond to questions, immature and perseverative, aggressive, overtly sexual or obscene in language, hyperphagic, and hypersomnolent, with occasional reports of headaches. Physical and neurological examinations were otherwise normal, apart from a small sebaceous naevus on the left side of his neck.

Spinal fluid examination was normal, including lactate and oligoclonal bands, although the opening pressure was slightly increased at $19 \mathrm{~cm}$ cerebrospinal fluid. Further biochemical, metabolic, and toxicological tests, and magnetic resonance imaging of the brain with gadolinium enhancement and special views of the hypothalamus and pituitary region were normal. The erythrocyte sedimentation rate on one occasion was $19 \mathrm{~mm} /$ hour and subsequently decreased to $9 \mathrm{~mm} /$ hour. A standard electroencephalogram (EEG) showed a mild excess of underlying slow activity at the time of one of the patient's typical episodes.

On further consideration it was felt that the clue to the correct diagnosis lay in the patient's hypersomnia (although this had been overshadowed by the behavioural disturbance). Polysomnography was performed during a typical episode. The patient had taken no recent drugs. The recording was performed on a hospital ward with unavoidable repeated interruptions for the patient's other special investigations. Even so, actual sleep time at night was nine hours 24 minutes compared with a normal 8-9 year old value of eight hours 34 minutes (SD 39 minutes). ${ }^{2}$ Sleep onset latency was nine minutes (mean (SD) 25 (16) minutes) and the rapid eye movement (REM) latency was 20.5 minutes (mean (SD) 129 (32) minutes). In addition, the patient slept a further two hours 30 minutes during the day, making a total of 11 hours 54 minutes in 24 hours. There was no reduction of stages 3 and 4 non-REM sleep. Although frequent brief arousals were seen during sleep, sleep architecture and sleep cycles remained basically intact and no seizure discharge was 
seen at any stage. Similar results were obtained in the second 24 hour period of polysomnography. Repeated sleep studies two months later and five days after the end of a disturbed episode produced normal results.

Treatment was initiated with clomipramine, which was ineffective at the maximum dose. After this, lithium was started and blood concentrations were monitored. There was a rapid response and the boy has remained normal since starting this treatment. He has been well for one year and withdrawal of lithium treatment is currently under consideration.

\section{Discussion}

Hypersomnia, defined as excessive sleep sufficient to seriously interfere with usual daytime activities, can be classified as persistent or recurrent. Occasionally, it is simulated for psychological reasons. There are a number of causes for hypersomnia, only some of which produce the recurrent forms. These include substance misuse, some endocrine and metabolic disorders, non-convulsive status epilepticus, menstrual associated hypersomnia, and the Kleine-Levin syndrome. Usually, severe depressive disorders are also listed although the degree of hypersomnia is modest.

The present patient's hypersomnia was genuine and recurrent with no evidence of causes other than the Kleine-Levin syndrome. The clinical features of hypersomnia, hyperphagia, and behavioural disturbance (including sexual disinhibition) make up the triad described by Critchley and Hoffman in 1942 as characteristic, in the absence of other medical or psychiatric disorders, of the KleineLevin syndrome. ${ }^{3}$

The diagnosis of this disorder is primarily clinical but, from the limited number of patients in whom physiological sleep studies have been performed, additional features can be objective confirmation of an increase in total sleep time to 12-14 hours or more in each 24 hours, reduced sleep latency and REM latency, and reduction in stages 3 and 4 of non-REM sleep. ${ }^{4}$ An EEG during an episode often shows non-specific generalised slowing of basic rhythms. The results of this patient's physiological sleep studies were compatible with this diagnosis, although the age of onset was younger than that usually reported in boys (median 16 years).

Two aspects of the disorder which, as in this instance, can increase the risk that it will be mistaken for a primarily psychological disorder are the intermittent nature of the disturbance and the often striking nature of the behavioural abnormalities.

In this patient, the mother's belief that the onset of his disturbed behaviour was somehow associated with his repeated anaesthetics was originally discounted because the disturbance was not persistent as might be expected with an organic cause. An explanation in terms of family factors was thought to be more likely. In fact, the onset of the syndrome is said to be preceded by a physical disturbance in $50 \%$ of patients, usually a flu-like illness or upper respiratory tract infection. ${ }^{5}$ Other physical factors reported as precipitating the disorder include head trauma, ${ }^{6}$ acute viral encephalitis, ${ }^{7}$ and (of particular interest in this patient) dental anaesthesia. ${ }^{8}$ It is also noteworthy that, although the illness in this child was fluctuating in the initial phase, the characteristic periodic pattern was not apparent until some weeks later - a further aspect of the clinical presentation which may render early diagnosis difficult.

The striking and often mixed behavioural abnormality in the classical form of the disorder can easily suggest a primarily psychological explanation for the symptoms and signs. Although the disturbed behaviour is out of character for the child, return to normality in between the episodes can be erroneously seen as further evidence for a non-organic process. The range of disturbed behaviours reported is wide, encompassing features suggestive of at least mild clouding of consciousness (disorientation), mood disorder (dysphoria, irritability, impulsiveness, depression, elation), overarousal (restlessness), disinhibition (aggression, sexual hyperactivity), or detachment from reality ('autistic' features, hallucinations).

The bizarre nature of many of the behaviours has been remarked on by some workers and may be an alerting sign. This was certainly a feature in this patient when strange or uncharacteristic behaviour included chasing a friend with a carving knife, stealing a cucumber from the grocers, hitting a woman in the street with a bag, swimming in his underpants, repeatedly changing the position of ornaments at home, putting an electric whisk in his mouth, and using foul language. Ferguson emphasised the bizarre quality of the behaviour of his patient, a 10 year old boy whose Kleine-Levin syndrome had been misdiagnosed as depression. ${ }^{8}$ Gillberg has made the same point in describing an adolescent whose disorder was also recognised only after a long delay, largely because of the lack of familiarity with the disorder by doctors and consequent preoccupation with other explanations of his strange behaviour. ${ }^{9}$

Available evidence suggest that the KleineLevin syndrome usually runs a benign course with spontaneous improvement over several years. In the meantime, drug treatment may be appropriate for individual attacks or prophylaxis. As the effect of stimulant drugs is shortlasting, they seem best reserved for patients in whom the episodes of hypersomnia are frequent. For the prevention of further attacks, tricyclic drugs and lithium are reported to be effective, but not in every patient, though some claim the latter as the undisputed treatment of choice. ${ }^{10}$ Although a good response to lithium is not invariable, it appears to have produced a convincing improvement in this child.

The authors are grateful to Dr David Jones for his opinion on psychiatric aspects of this case and Dr Zenobia Zaiwalla for the neurophysiological recordings.

\footnotetext{
1 Billiard M. Other hypersomnias. In: Thorpy MJ, ed. Handbook of sleep disorders. New York: Marcel Dekker, 1990: 353-71.

2 Coble PA, Kupfer DJ, Reynolds III CF, Houck P. EEG sleep of healthy children 6 to 12 years of age. In:
} 
Guilleminault $\mathrm{C}$, ed. Sleep and its disorders in children. New York: Raven Press, 1987: 29-41.

3 Critchley M, Hoffman HL. The syndrome of periodic somnolence and morbid hunger (Kleine-Levin syndrome). BMF 1942; i: 137-9.

4 Diagnostic Classification Steering Committee, Thorpy MJ, chairman. International classification of sleep disorders diagnostic and coding manual. Rochester: American Sleep Disorders Association, 1990: 43-6. 5 Billiard M, Cadilhac J. Les hypersomnies recurrentes. Rev

6 Will RG, Young JPR, Thomas DJ. Kleine-Levin syn- drome: report of two cases with onset of symptoms precipitated by head trauma. Br f Psychiatry 1988; 152 410-2.

7 Merriam AE. Kleine-Levin syndrome following acute viral encephalitis. Biol Psychiatry 1986; 21: 1301-4.

Ferguson BG. Kleine-Levin syndrome: a case report f Child Psychol Psychiatry 1986; 27: 275-8.

9 Gillberg C. Kleine-Levin syndrome: unrecognised diagnosis in adolescent psychiatry $7 \mathrm{Am}$ Acad Child Adolesc Psychiatry 1987; 26: 793-4.

10 Goldberg MA. The treatment of Kleine-Levin syndrome with lithium. Can $\mathcal{f}$ Psychiatry 1983; 28: 491-3.

\section{Down's syndrome}

Three papers in the July 1994 issue of Developmental Medicine and Child Neurology (1994; 36: 576-85, 586-93, and 594-600) describe problems encountered by children with Down's syndrome. It has been estimated that between 0.6 and $13 \%$ of children with Down's syndrome also have infantile spasms. Seventeen children with this combination were identified in Boston, Massachusetts (Carl E Stafstrom and Richard J Konkol: 576-85). In general the addition of infantile spasms to Down's syndrome seemed less disastrous than might have been expected. The prognosis for control of seizures was quite good and developmental progress on the whole remained within the range expected in Down's syndrome, although some children who regressed with the onset of infantile spasms remained developmentally impaired.

Workers in Newfoundland (Mary L Courage and colleagues: 586-93) measured visual acuity in 51 infants and children with Down's syndrome. They found a significant reduction in visual acuity and delayed development of visual acuity especially after the age of 6 months.

In Chicago 77 unselected children at a Down's syndrome clinic were examined first by a developmental paediatrician and then by a paediatric ophthalmologist (Nancy J Roizen and colleagues: 594-600). The paediatrician found an ophthalmic abnormality in 31 children and the ophthalmologist confirmed these and found an abnormality in a further 15 . Overall $61 \%$ of children had an ophthalmic abnormality, $38 \%$ of the infants and $80 \%$ of those aged 5 to 12 years. The most common disorders were refractive errors, squint, and nystagmus. These authors conclude that all children with Down's syndrome should be examined by a paediatric ophthalmologist before the age of 6 months and regularly after that. 